\title{
Exposure to Acrylamide in the Sixth Total Diet Study - China, 2016-2019
}

\author{
Feng Zhu'; Yuxin Wang, ${ }^{2, *}$; Hualiang Liu'; Bing Lyu'; Dawei Chen²; Jingguang $\mathrm{Li}^{2}$; \\ Wenliang Ji ${ }^{1, *}$; Yunfeng $\mathrm{Zhao}^{2}$; Yongning Wu
}

\section{Summary \\ What is already known about this topic?}

Acrylamide (AA) is toxic and potentially carcinogenic and could be formed during the cooking process. It is understood that almost all foods cooked at high temperature contain AA, especially fried foods. The exposure of AA in food threatens human health.

What is added by this report?

In the Sixth China Total Diet Study (TDS), AA was detected in $73.3 \%$ samples of the 12 food categories with the concentrations ranging from undetected to $176.90 \mu \mathrm{g} / \mathrm{kg}$. The average dietary intake of AA was $0.175 \mu \mathrm{g} / \mathrm{kg}$ body weight per day and a significant decrease $(45.1 \%)$ was observed compared with the Fifth China TDS. Among the 12 food categories, vegetables $(59.0 \%)$, cereals $(18.9 \%)$, and potatoes $(10.1 \%)$ were the main contributors to AA exposure at $88.0 \%$.

What are the implications for public health practice?

This study highlighted the need to continuously monitor dietary acrylamide exposure in China, including changing food processing methods and making reasonable selection of foodstuffs in the daily diet.

Acrylamide (AA) is widely used in dam and tunnel construction, paper manufacturing, the oil industry, cosmetics, and pharmaceuticals. It has been reported to be a rodent carcinogen (Group 2A) and a probable carcinogen to humans, possibly having toxic effects on the nervous system along with adverse reproductive and developmental effects. Although there is no limit standard for AA in food, relevant control measures have been implemented by several countries. In 2012, the China National Center for Food Safety Risk Assessment released the "Risk Assessment Report of Acrylamide in Food," which evaluated its toxicity, formation, and possible human exposure, and proposed suggestions for its control and prevention (1).
Figure 1 showed the contributions of 12 food categories to the overall dietary exposure as analyzed in the Sixth Total Diet Study (TDS), the methodology of which was presented in the Foreword of this issue (2). Vegetables $(59.0 \%)$ and cereals $(18.9 \%)$ were the predominant contributors of AA intake, accounting for approximately $80 \%$ of the overall estimated dietary intake (EDI). In addition, potatoes were also an important source of AA exposure (10.1\%).

Using mean EDI value $(0.175 \mu \mathrm{g} / \mathrm{kg}$ body weight per day) with the no observed adverse effect level (NOAEL) value $(0.2 \mathrm{mg} / \mathrm{kg}$ body weight per day), calculated margin of exposure (MOE) value was 1,142 . This result indicates the MOE was well below 10,000. Following the conclusions of European Food Safety Authority (EFSA) committee, a potential human health risk should be concerned.

\section{DISCUSSION}

In this study, an up-to-date AA database of the Sixth TDS was established to estimate AA exposure for Chinese adults. We found that cereals and vegetables were $100 \%$ contaminated with AA, while water and beverages and alcohol beverages had the lowest contamination rates (Table 1). The highest contamination level of AA was found in potatoes from Jiangxi $(176.90 \mu \mathrm{g} / \mathrm{kg})$, followed by the vegetables from Guizhou $(154.85 \mu \mathrm{g} / \mathrm{kg})$ and sugar from Hebei $(129.31 \mu \mathrm{g} / \mathrm{kg})$. Foods cooked at high temperatures (above $120{ }^{\circ} \mathrm{C}$ ) upon frying, roasting, and baking were found to produce large amounts of acrylamide, especially starchy foods such as potatoes. The mean concentration of potatoes was $17.74 \mu \mathrm{g} / \mathrm{kg}$, which was only half of our last TDS (3). It was lower than the mean level of non-fried products of potatoes $(108 \mu \mathrm{g} / \mathrm{kg})$ in the report of EFSA (4), and also much lower than the mean level of potato snacks $(554.5 \mu \mathrm{g} / \mathrm{kg})$ in a survey conducted in the Republic of Korea (5). According to the commission regulation European Union (EU) 2017/2158, the AA benchmark levels of potato products $(750 \mu \mathrm{g} / \mathrm{kg})$, barley and rice- 


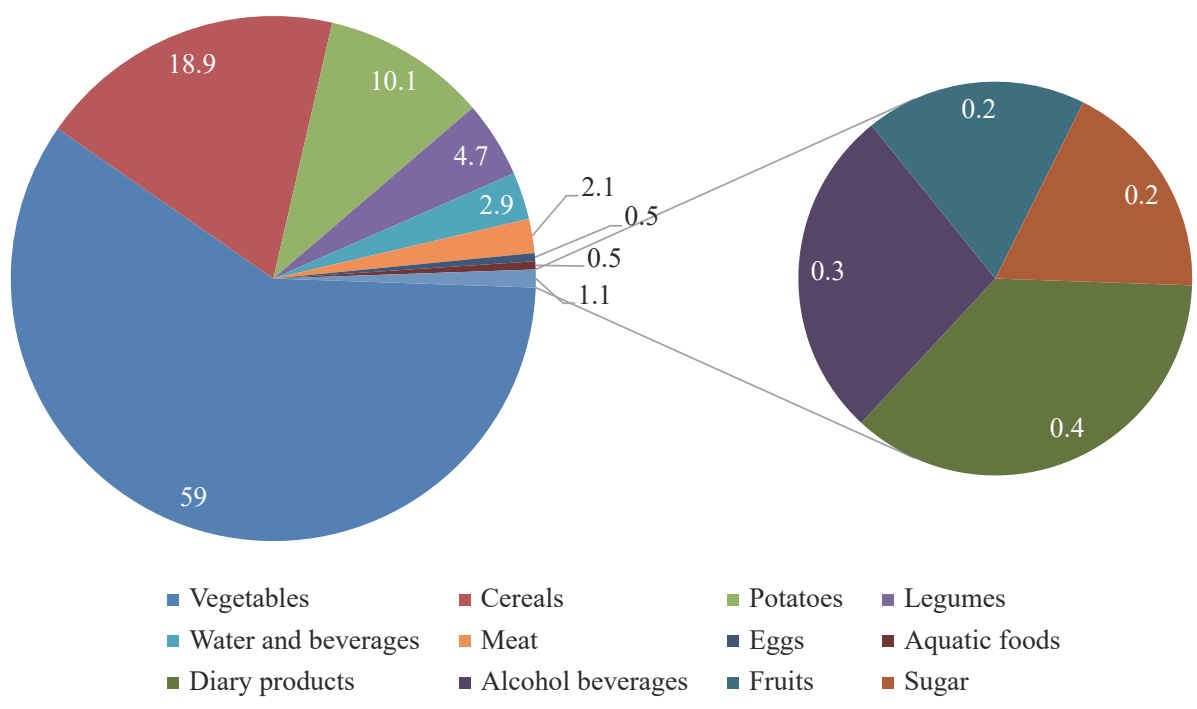

FIGURE 1. Daily intake of the different food groups to dietary acrylamide intakes for Chinese general population (\%).

based products $(150 \mu \mathrm{g} / \mathrm{kg})$, roast coffee $(400 \mu \mathrm{g} / \mathrm{kg})$, and baby foods $(40 \mu \mathrm{g} / \mathrm{kg})$ were reported, which were above levels found in the Sixth TDS. Due to the differences in eating habits, cooking methods, heating temperature, and amount of amino acids and carbohydrates, the levels of AA in the same food category varied between different provincial-level administrative divisions (PLADs).

The main contributing food group based on the Sixth TDS results was vegetables, followed by cereals, potatoes, legumes and nuts, water and beverages and meat, which was consistent with the Fifth TDS results but different from other international studies. For instance, Japan's main contributor of AA exposure was beverages, followed by confectioneries, vegetables, potatoes and starches, and cereals (G). While for EU countries, the main contributors were potato fries, followed by bread, other potato products, biscuits, crackers and crispbreads, coffee, and other cereal products $(7)$.

The dietary exposure to AA in the Third, Fourth, Fifth, and Sixth TDSs was 0.188, 0.286, 0.319, and $0.175 \mu \mathrm{g} / \mathrm{kg}$ body weight per day, respectively (2). The mean EDIs of AA for the previous 3 studies showed a significant increasing trend, while a significant decrease of $45.1 \%$ (compared with the Fifth TDS) was observed in this study, and the values were the lowest since 2000. According to the 4 TDS results, vegetables were consistently the main contributor of AA intake, and the results were all relatively stable with more than 0.1 $\mu \mathrm{g} / \mathrm{kg}$ body weight per day, followed by cereals, potatoes, and legumes (Table 2). The EDI of cereals decreased significantly compared with the previous three times, resulting in the lowest mean dietary exposure to AA in the Sixth TDS. Furthermore, the EDIs of individual PLADs were compared between the Fourth, Fifth, and Sixth Chinese TDS (Figure 2). The EDIs varied in different PLADs, but fortunately, half of the PLADs' EDI values decreased to the lowest level from 2005 to 2019. For some areas, such as Heilongjiang, Hebei, and Hubei, the main contributors (cereals) decreased compared with the previous study, resulting in a dramatic drop of TDS in these PLADs. The value obtained in the current study was much lower than most international studies $(3,6,8)$, which indicated that the national dietary exposure risk to $\mathrm{AA}$ is at a low level compared to the world.

The MOE approach was employed to estimate the risk of AA exposure by Joint Expert Committee for Food Additives (JECFA) in 2021. Based on the NOAEL value for morphological nerve changes (0.2 $\mathrm{mg} / \mathrm{kg}$ body weight per day), the overall Chinese MOE of the Sixth TDS was 1,142. According to the conclusions of EFSA committee, an MOE value of 10,000 or higher would be of low public health concern. Hence, the current value $(1,142)$ is far below 10,000 , indicating a potential human health risk that should be of concern for Chinese population. Notably, the MOE value for Guizhou was very low (191), which was lower than the value (310) reported by JECFA. Therefore, efforts should be made to reduce dietary exposure to AA, both by changing food processing methods and promoting healthy eating habits. However, there are several limitations of this study, such as food coverage, food processing method 


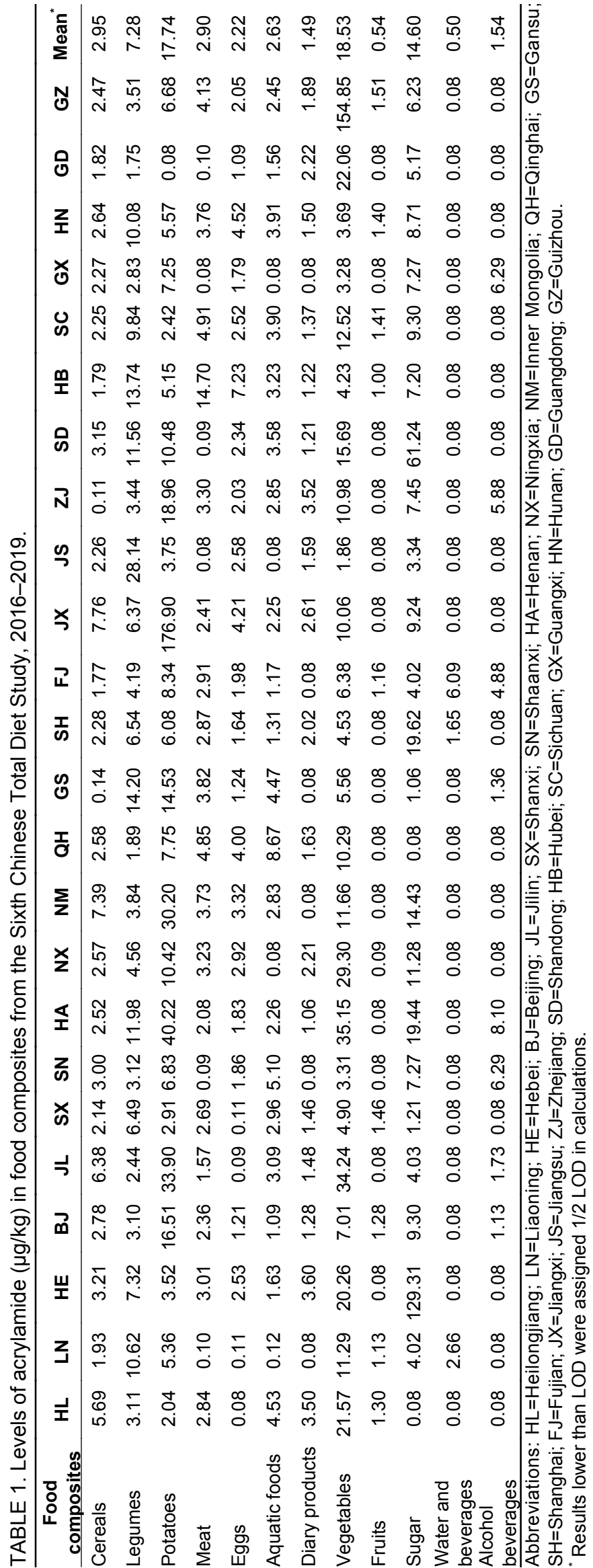

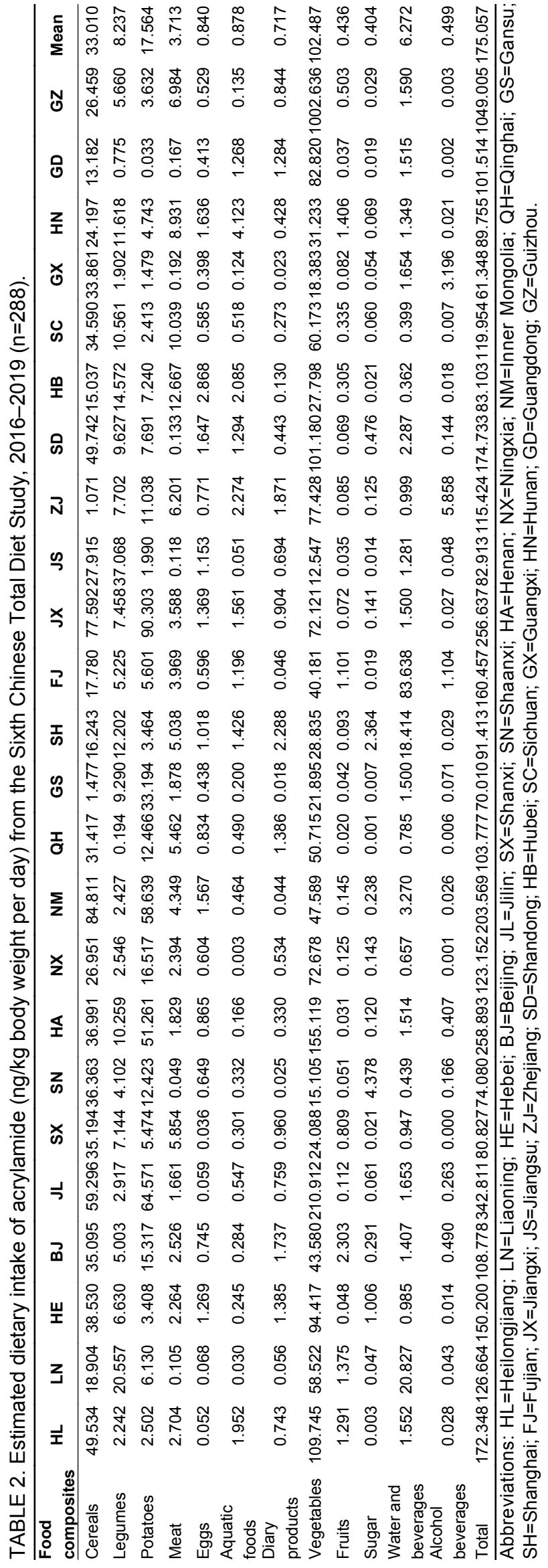




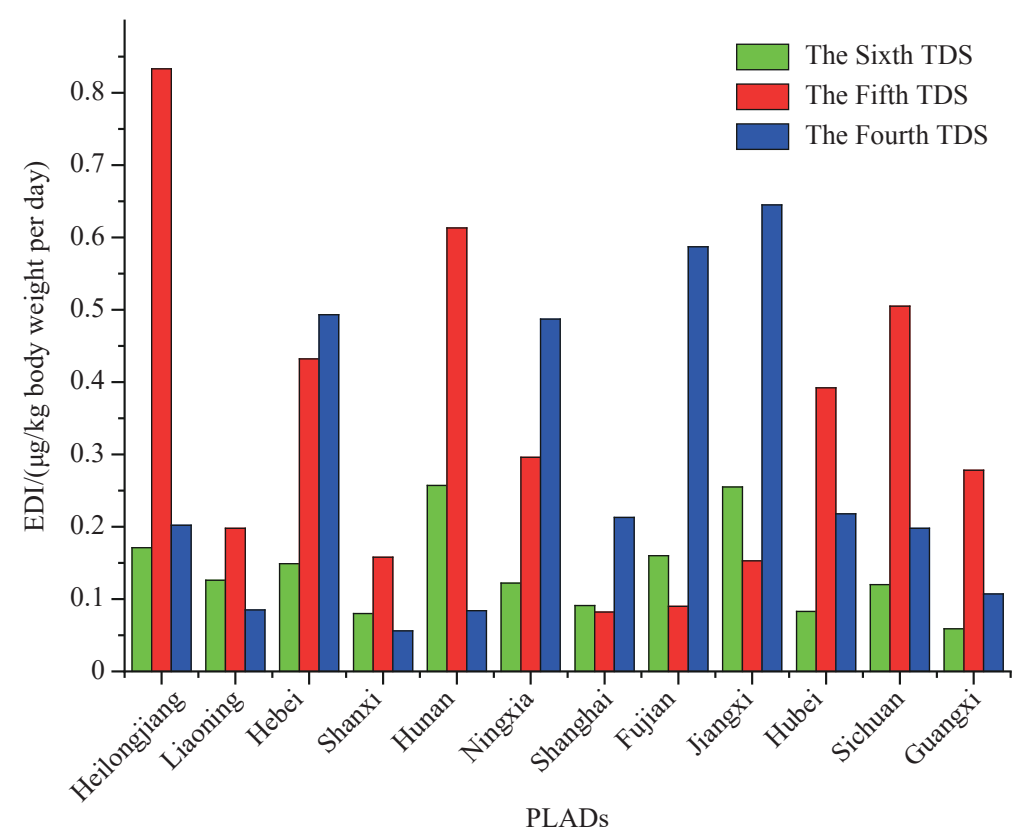

FIGURE 2. Comparative estimated daily intakes of acrylamide from the Fourth, Fifth, and Sixth TDS in various PLADs in China.

Abbreviations: TDS=Total Diet Study; PLADs=provincial-level administrative divisions; EDI=estimated dietary intakes.

(temperature, time, etc.), uncertainties of consumption data, accuracy of detection method, and no assessment for different ages.

In the Sixth TDS, the mean EDI of AA for the Chinese population was $0.175 \mu \mathrm{g} / \mathrm{kg}$ body weight per day, which was lower than the values of the three previous TDSs and other international studies, indicating that measures implemented to reduce AA were effective from 2016 to 2019. However, the calculated MOE value was relatively low, still implying potential human health concerns. Vegetables, cereals, and potatoes were the 3 top predominant sources and accounted for $88 \%$ dietary exposure to $\mathrm{AA}$. It is necessary to monitor continuously the AA exposure risk in the Chinese population. In the future, food processing methods and daily diet should be altered to reduce dietary exposure to acrylamide.

Conflicts of interest: No conflicts of interest.

Acknowledgements: The 24 provincial-level CDCs in China.

Funding: Supported by the National Key Research and Development Program of China (NO.2017YFC 1600500), and CAMS Innovation Fund for Medical Science (CIFMS 2019-I2M-5-024).

doi: $10.46234 / \mathrm{ccdcw} 2022.040$

\# Corresponding authors: Yuxin Wang, wangyx@cfsa.net.cn; Wenliang Ji,641827872@qq.com.

\footnotetext{
Jiangsu Provincial Center for Disease Control and Prevention, Nanjing, Jiangsu Province, China; ${ }^{2}$ NHC Key Laboratory of Food
}

Safety Risk Assessment, Chinese Academy of Medical Science Research Unit (No. 2019RU014), China National Center for Food Safety Risk Assessment, Beijing, China.

Submitted: November 18, 2021; Accepted: February 26, 2022

\section{REFERENCES}

1. China National Center for Food Safety Risk Assessment. Risk assessment report of aacrylamide in food. 2012. https://www.cfsa.net.cn/Article/ News.aspx? $\mathrm{id}=17$ A52A7320B33FBD9E43894CFB89B7D91B8FAB 82336415E487D226792ABB6E1A365F8A2D46F94D3E. [2021-1117]. (in Chinese).

2. Lyu B, Li JG, Wu YN. Characterizing exposome of food contamination and China Total Diet Study: project for improving food safety risk assessment in China. China CDC Wkly 2022;4(9):157 - 60. http://dx. doi.org/10.46234/ccdcw2022.039.

3. Gao J, Zhao YF, Zhu F, Ma YJ, Li XW, Miao H, et al. Dietary exposure of acrylamide from the fifth Chinese Total Diet Study. Food Chem Toxicol 2016;87:97 - 102. http://dx.doi.org/10.1016/j.fct.2015.11.013.

4. EFSA Panel on Contaminants in the Food Chain (CONTAM). Scientific opinion on acrylamide in food. EFSA J 2015;13(6):4104. http: //dx.doi.org/10.2903/j.efsa.2015.4104.

5. Lee S, Kim HJ. Dietary exposure to acrylamide and associated health risks for the Korean population. Int J Environ Res Public Health 2020;17(20):7619. http://dx.doi.org/10.3390/ijerph17207619.

6. Kotemori A, Ishihara J, Nakadate M, Sawada N, Iwasaki M, Sobue T, et al. Validity of a self-administered food frequency questionnaire for the estimation of acrylamide intake in the Japanese population: the JPHC FFQ Validation Study. J Epidemiol 2018;28(12):482 - 7. http://dx.doi. org/10.2188/jea.JE20170186.

7. Raffan S, Halford NG. Acrylamide in food: progress in and prospects for genetic and agronomic solutions. Ann Appl Biol 2019;175(3):259 - 81 http://dx.doi.org/10.1111/aab.12536.

8. Wong WWK, Chung SWC, Lam CH, Ho YY, Xiao Y. Dietary exposure of Hong Kong adults to acrylamide: results of the first Hong Kong Total Diet Study. Food Addit Contam Part A 2014;31(5):799 - 805. http:// dx.doi.org/10.1080/19440049.2014.898189. 